\title{
Design and Calibration of an RF Actuator for Low Level RF Systems
}

\author{
Zheqiao Geng, Member, IEEE, Bo Hong
}

\begin{abstract}
X-ray Free Electron Laser (FEL) machines like the Linac Coherent Light Source (LCLS) at SLAC require high quality electron beams to generate $x$-ray lasers for various experiments. Digital Low Level RF (LLRF) systems are widely used to control the high power RF klystrons to provide highly stable RF field in accelerator structures for beam acceleration. Feedback and feed forward controllers are implemented in LLRF systems to stabilize or adjust the phase and amplitude of the RF field. To achieve the RF stability and the accuracy of the phase and amplitude adjustment, low noise and highly linear RF actuators are required. Aiming for the upgrade of the S-band Linac at SLAC, an RF actuator is designed with an I/Q modulator driven by two Digital-to-Analog Converters (DAC) for the digital LLRF systems. Direct up-conversion scheme is selected for RF actuation and an on-line calibration algorithm is developed to compensate the $\mathrm{RF}$ reference leakage and the imbalance errors in the $I / Q$ modulator which may cause significant phase and amplitude actuation errors. This paper presents the requirements on the $\mathrm{RF}$ actuator, the design of the hardware, the calibration algorithm and the implementation in firmware and software and the test results at LCLS.
\end{abstract}

Index Terms-LLRF, RF Actuator, I/Q Modulator, I/Q Imbalance, Direct Up-conversion

\section{INTRODUCTION}

L CLS [1] and the Facility for Advanced Accelerator Experimental Tests (FACET) [2] are two user facilities in operation based on the existing 2-mile S-band (2856 MHz) linear accelerator [3] at SLAC National Accelerator Laboratory. A digital LLRF system with the block diagram shown in Fig. 1 is proposed aiming for the upgrade of the obsolete LLRF systems of the Linac. It consists of multichannel RF signal detectors for phase and amplitude measurements, FPGA controllers for feedback and feed forward control and RF actuators for RF pulse generation and phase and amplitude adjustments.

The LLRF system should provide high RF stabilities in accelerator structures considering the tightest requirements for

Manuscript received August 03, 2015.

Z. Geng was with the Instrumentation and Controls Division, SLAC National Accelerator Laboratory, Menlo Park, CA 94025 USA. He is now with the LLRF section, Paul Scherrer Institut, 5232 Villigen PSI, Switzerland (phone: +41 7886380 59; e-mail: zheqiao.geng@psi.ch).

B. Hong is with the Instrumentation and Controls Division, SLAC National Accelerator Laboratory, Menlo Park, CA 94025 USA (email: hongbo@slac.stanford.edu).
LCLS, which requests the phase stability to be better than $0.1^{\circ}$ (RMS) and amplitude stability better than $0.1 \%$ (RMS) [4].

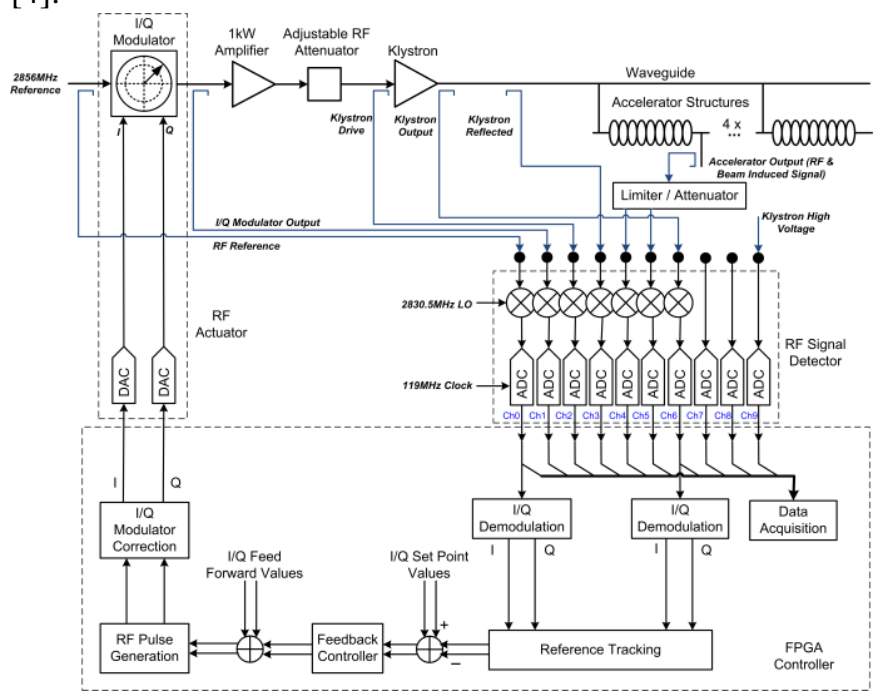

Fig. 1. Block diagram of the digital LLRF system for an RF station.

The LCLS Linac is based on normal conducting travelling wave accelerator structures working at a repetition rate of 120 $\mathrm{Hz}$. The length of RF pulses is too short $(\sim 3.5 \mu \mathrm{s})$ to utilize intra-pulse feedbacks for RF stability control. A pulse-to-pulse feedback controller will be implemented to reduce the slow fluctuations in the RF field. To study the transfers of driving chain noise and measurement noise to the RF field, a simplified block diagram of the feedback loop is depicted in Fig. 2. The RF system is modeled as a static system with its system gain normalized to 1 for pulse-to-pulse behaviors, and a discrete integral controller will be adopted.

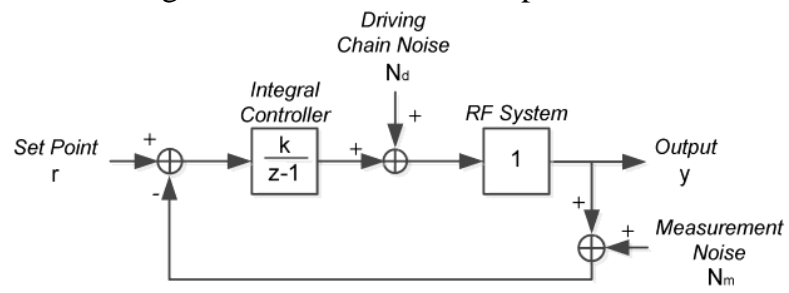

Fig. 2. Pulse-to-pulse feedback loop for an RF station. The transfer function of the controller is presented by Z-transform with the integral gain of $\mathrm{k}$. In the block diagram, $r$ represents the amplitude and phase set points, $y$ represents the RF field for beam acceleration, $N_{d}$ is the driving chain noise and $\mathrm{N}_{\mathrm{m}}$ is the measurement noise.

The driving chain noise mainly comes from the RF actuator, $1 \mathrm{~kW}$ amplifier and klystron modulator. One of the major noise sources for the RF actuator is the DAC power supply. A 
low noise amplifier should be selected to reduce the $1 / \mathrm{f}$ noise and thermal noise added by it [5]. The jitters in the klystron modulator high voltage are the most critical source of driving chain noise. The measurement noise mainly comes from the down converters and ADCs. Thermal drifts exist at anywhere in the driving chain and measurement chain when temperature changes. The RF jitters caused by the driving chain and measurement noises will be aliased to the first Nyquist bandwidth of the $120 \mathrm{~Hz}$ repetition rate, resulting in the pulseto-pulse jitters (e.g. noises at frequencies of integer times of $120 \mathrm{~Hz}$ will be aliased to $0 \mathrm{~Hz}$ in the pulse-to-pulse jitter measurement).

The transfers of driving chain noise and measurement noise to the RF field in frequency domain in the Nyquist bandwidth of the machine repetition rate are depicted in Fig. 3.

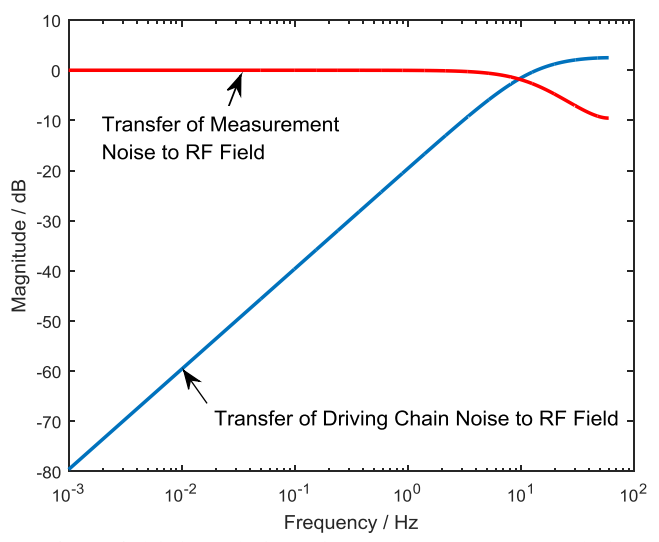

Fig. 3. Transfers of driving chain noise and measurement chain noise to the $\mathrm{RF}$ field with integral gain $\mathrm{k}=0.5$. The driving chain noise slower than 7.8 $\mathrm{Hz}$ can be suppressed by more than $-3 \mathrm{~dB}$, while the driving chain noise faster than $10 \mathrm{~Hz}$ will be amplified by up to $2.5 \mathrm{~dB}$.

To suppress the fast jitters in the driving chain, like the jitters caused by klystron modulator high voltage, the integral gain has to be increased with the cost to further amplify the high frequency driving chain noise and to pass more measurement noise into the RF field. During normal operation, the integral gain is usually set low to avoid increasing the pulse-to-pulse jitters but still be able to remove the slow drifts in the RF system. With this consideration, the bandwidth to suppress the driving chain noise should not be expected to be larger than several $\mathrm{Hz}$. To achieve the required short-term RF stabilities in accelerator structures, low noise driving chain components are essential; while to achieve good long-term stabilities, low drift RF measurement electronics will be required.

The slow drifts in the RF driving chain will be suppressed by the pulse-to-pulse feedbacks, while the fast jitters will be determined by the diving chain components themselves and can be estimated in equation (1), assuming the contributions by different components are not correlated with each other:

$$
N_{D R V}=\sqrt{N_{A C T}^{2}+N_{A M P}^{2}+N_{K L Y}^{2}},
$$

where $N_{D R V}$ is the overall phase or amplitude jitters caused by the driving chain, $N_{A C T}, N_{A M P}$ and $N_{K L Y}$ stand for the jitters contributed by the RF actuator, $1 \mathrm{~kW}$ amplifier and klystron respectively with the closed-loop transfer function in Fig. 3 taken into account. For the RF stations in LCLS injector operated in linear range, equation (1) is valid for both the amplitude and phase jitters; while for most RF stations in LCLS main Linac operated in saturation, the amplitude jitters of the RF actuator and $1 \mathrm{~kW}$ amplifier are not critical [6]. In order to achieve the final RF stabilities, a low noise RF actuator is very helpful to relax the stability requirements on high power parts which are more costly to reduce jitters.

The RF actuator consists of an I/Q modulator and two DACs. Controlled by the FPGA, the RF actuator can perform fast phase and amplitude actuations with complex pulse shapes. The major requirements to the RF actuator are summarized below:

1) The RF actuator should be able to generate RF pulses with arbitrary phase and amplitude modulations.

2) The additive phase jitter and amplitude jitter caused by the RF actuator should be smaller than $0.01^{\circ}$ (RMS) and $0.01 \%$ (RMS) respectively within the beam interacting bandwidth (around $450 \mathrm{kHz}$ ) of the accelerator structures.

3) The phase and amplitude actuation accuracy should be better than $\pm 1^{\circ}$ and $\pm 1 \%$ respectively when rotating the phase setting for a full cycle while keeping the amplitude setting constant or increasing the DAC output from 50\% to full scale while keeping the phase setting constant.

An I/Q modulator module was designed and a calibration algorithm was developed for the LLRF system to satisfy the requirements shown above.

\section{RF ACTUATOR DESIGN}

\section{A. Hardware Design}

An I/Q modulator module was implemented with custom designed Printed Circuit Board (PCB) and surface-mount RF components like $0^{\circ}$ and $90^{\circ} \mathrm{RF}$ power splitter/combiners, mixers and RF amplifiers. The block diagram and the picture of the I/Q modulator module are shown in Fig. 4 and Fig. 5.

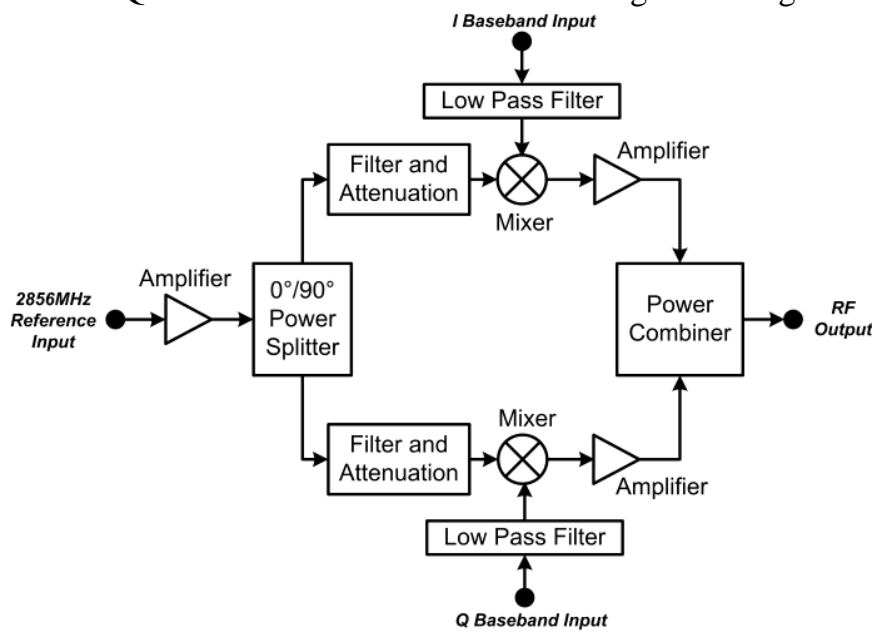

Fig. 4. Block diagram of the $\mathrm{I} / \mathrm{Q}$ modulator module. 


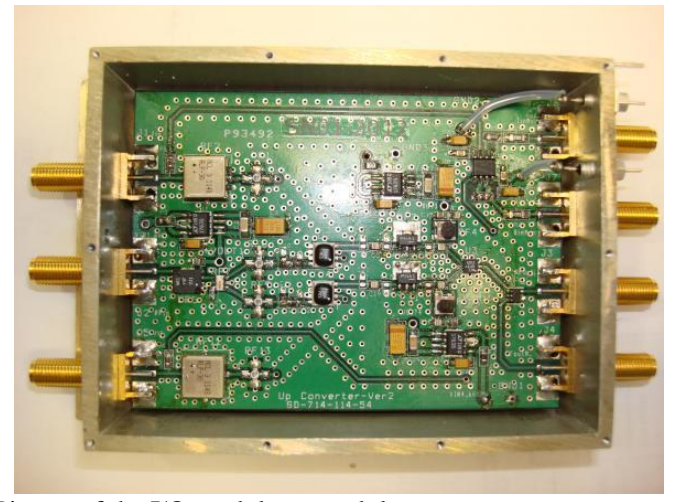

Fig. 5. Picture of the I/Q modulator module.

\section{B. Up-conversion Algorithm Selection}

With the configuration of dual DACs and an I/Q modulator, both single side band (SSB) up-conversion and direct upconversion [7] algorithms can be implemented.

For the SSB up-conversion scheme, the local oscillator (LO, $2830.5 \mathrm{MHz}$ ) signal is used as the reference input to the I/Q modulator and the DACs are used to generate intermediate frequency (IF, $25.5 \mathrm{MHz}$ ) signals with modulated phase and amplitude for the baseband input ports. The phases of the IF signals from the two DACs are separated by $90^{\circ}$ to pick up the upper sideband $(2856 \mathrm{MHz})$ of the mixer output. With the SSB up-conversion scheme, the low frequency noises (around several hundred $\mathrm{kHz}$ ) in the DAC outputs which are mostly caused by the noises in the power supplies can be easily filtered out with a band-pass filter on the RF output. But an interruption of the DAC clock will result in an arbitrary phase in the IF signal after the clock recovers. With the SSB upconversion scheme, a robust clock and a smart clock synchronization scheme are required to keep the proper phase of the IF signal, which will increase the complexity of the RF actuator.

For the direct up-conversion scheme, the reference signal $(2856 \mathrm{MHz})$ is used as the input to the I/Q modulator and the DACs are used to generate baseband signals close to square wave pulses. With this scheme, the phase will be kept after a cycle for the clock to be interrupted and recovered and the race conditions between the trigger and clock will not be a critical issue. The RF actuator design will be simpler resulting in higher reliability. But the noises of the power supplies within the bandwidth of the RF system become more critical and the RF actuator should be electromagnetic compatible not to pick up noises from the environment. Another issue for the direct up-conversion scheme is that the RF reference leakage and imbalances in $\mathrm{I} / \mathrm{Q}$ modulator will generate phase and amplitude actuation errors in the RF output.

For the digital LLRF system at SLAC, the direct upconversion scheme is finally selected with a careful selection of the power supply for the DACs.

\section{RF ACTUATOR CALIBRATION}

\section{A. Use Cases of RF Actuator}

From the operation experiences of LCLS and FACET, the
RF actuators are used in the following cases:

1) Adjust the amplitude and phase of the RF station without changing the shape of RF pulses. In this case, the phase and amplitude actuation errors can also be compensated by pulse-to-pulse feedbacks.

2) Generate frequency offset in RF output with linear phase variations within RF pulses. This may be used in the application to measure the frequency response of high power RF components [8]. There was also once a special request to operate one RF station at slightly different frequency to compensate the errors in the manufacture of accelerator structures. In this case, the RF reference leakage and imbalances in I/Q modulator should be compensated to achieve accurate phase variations and constant amplitude within RF pulses. In frequency domain, this is equivalent to an SSB operation mode which requires the carrier frequency and image frequency signals to be well suppressed.

In general, if accurate pulse shapes are required when actuating on the RF phase and amplitude, it will be necessary to calibrate the I/Q modulator in addition to the pulse-to-pulse feedbacks. I/Q modulators have been widely used in accelerator controls [9-10] and lots of calibration algorithms have been developed [11-14]. Most of the existing calibration algorithms either require special setups or need to interrupt the normal operation. In this paper, an analytical error model of the I/Q modulator will be established and a calibration algorithm will be derived from it. An on-line calibration scheme without interrupting the normal operation will be worked out. The calibration will be carried out iteratively to be able to track the drifts in RF actuator.

\section{B. Error Model of I/Q Modulator}

The phase and amplitude imbalances of the I/Q modulator are mainly caused by the imperfection of the RF components and the PCB fabrication. The imbalances are mostly system errors but may also change with the environment temperature and input power level.

The phase and amplitude imbalances and the reference leakage will cause phase and amplitude actuation errors which are depicted in Fig. 6 [15].

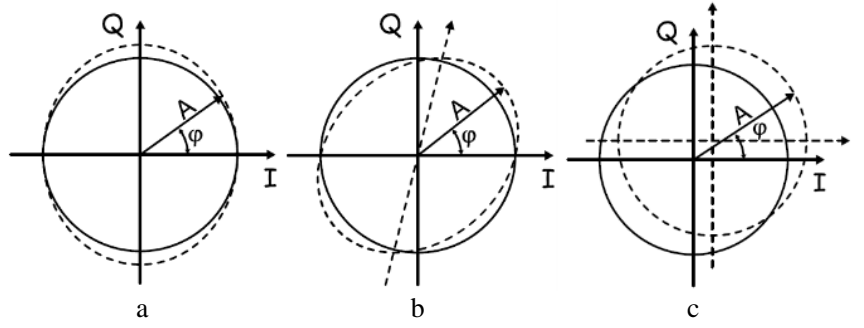

Fig. 6. RF actuation errors caused by the phase and amplitude imbalances of the $\mathrm{I} / \mathrm{Q}$ modulator and the reference leakage.

a. RF actuation errors caused by $\mathrm{I} / \mathrm{Q}$ modulator amplitude imbalance

b. RF actuation errors caused by I/Q modulator phase imbalance

c. RF actuation errors caused by reference leakage

The leakage of the RF reference can be compensated by introducing proper offsets in the two DAC channels. The 
offsets generate an extra RF output with the same amplitude but opposite phase compared to the reference leakage, resulting in cancellation of the leakage signal.

In the LLRF system, the output of the I/Q modulator will be measured by an RF detector to evaluate the errors in the RF actuator. The RF detector contains a down converter to convert the RF signal to IF frequency $(25.5 \mathrm{MHz})$ which is sampled by a 16-bit ADC with a clock frequency of $119 \mathrm{MHz}$. The non-IQ demodulation algorithm [16] averaged with 14 samples covering 3 IF periods is used to calculate the amplitude and phase of the RF signal. The RF detector electronics have a great resolution (about 5e-5 RMS and $0.005^{\circ} \mathrm{RMS}$ for amplitude and phase respectively within the bandwidth of accelerator structures interacting with beam). The third-order intercept point (IP3) of input power to the RF detector is around $40 \mathrm{dBm}$. By limiting the maximum RF input power to $13 \mathrm{dBm}$, which can still generate an IF amplitude around $0.9 \mathrm{~V}$ into the $\mathrm{ADC}$, the amplitude and phase measurement errors caused by the non-linearity of RF detector will be smaller than $0.2 \%$ and $0.12^{\circ}$ respectively [15]. The accuracy of RF measurement channels is enough for RF actuator calibration and on the other hand, they also determine the performance limits for the calibration.

Considering the DAC offsets, the imbalances of the I/Q modulator, the demodulation algorithm and the arbitrary cabling, the error model of the RF actuator can be depicted as in Fig. 7. The parameters in the model are described below.

$$
\text { TABLE I }
$$

PARAMETERS OF ERROR MODEL FOR RF ACTUATOR

\begin{tabular}{ll}
\hline \hline \multicolumn{1}{c}{ Parameter } & \multicolumn{1}{c}{ Description } \\
\hline $\mathrm{I}_{\mathrm{off}}$ & Offset of DAC for I channel \\
$\mathrm{Q}_{\text {off }}$ & Offset of DAC for Q channel \\
$\mathrm{g}$ & Amplitude imbalance \\
$\varphi$ & Phase imbalance \\
$\mathrm{K}_{\mathrm{i}}+\mathrm{j} \mathrm{K}_{\mathrm{q}}$ & Complex coefficients to model the attenuation \\
& and phase shift caused by cables \\
\hline \hline
\end{tabular}

From the error model, we can find an analytical description between the DAC settings and the output of the I/Q modulator. With the measured results from the I/Q modulator with different DAC settings, the parameters listed in Table I can be derived by linear fittings, which can be used to decide the correction coefficients in FPGA.

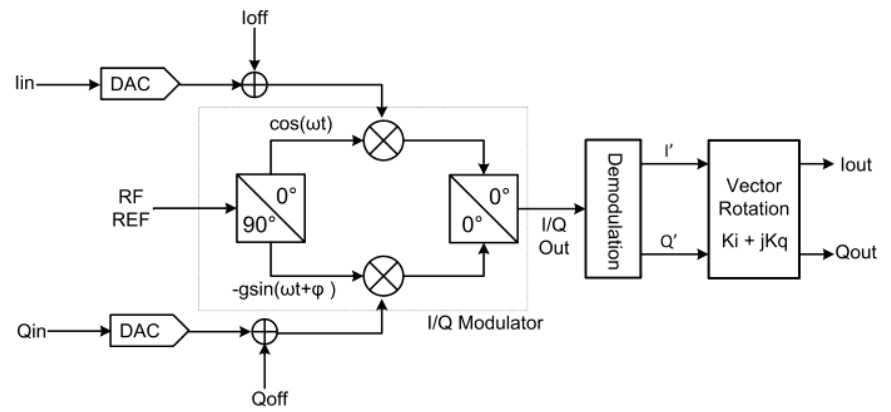

Fig. 7. Error model of the RF actuator.

For the model in Fig. 7, the relations between the output and input can be described by a first order equation:

$$
\left[\begin{array}{l}
I_{\text {out }} \\
Q_{\text {out }}
\end{array}\right]=\left[\begin{array}{ll}
a & b \\
c & d
\end{array}\right]\left[\begin{array}{l}
I_{\text {in }} \\
Q_{\text {in }}
\end{array}\right]+\left[\begin{array}{l}
e \\
f
\end{array}\right] .
$$

where

$$
\begin{aligned}
& a=K_{i}, \quad c=K_{q} \\
& b=-g\left(K_{i} \sin \varphi+K_{q} \cos \varphi\right) \\
& d=g\left(K_{i} \cos \varphi-K_{q} \sin \varphi\right) \\
& e=a I_{o f f}+b Q_{o f f}, \quad f=c I_{o f f}+d Q_{o f f}
\end{aligned}
$$

The DAC settings (Iin and Qin) can be scanned for a full circle in several steps with fixed amplitude and rotating phases and the output of the I/Q modulator (Iout and Qout) can be measured for each step. The parameters of $a$ to $f$ can be calculated by linear fitting and the parameters in the error model can be derived from equation (3) as

$$
\begin{aligned}
& K_{i}=a, K_{q}=c \\
& I_{o f f}=\frac{b f-d e}{b c-a d}, \quad Q_{\text {off }}=\frac{c e-a f}{b c-a d} . \\
& g=\sqrt{\frac{b^{2}+d^{2}}{a^{2}+c^{2}}}, \quad \tan \varphi=\frac{a b+c d}{b c-a d}
\end{aligned}
$$

The parameters in equation (4) will be used to correct the errors in the RF actuator.

\section{Corrections of I/Q Modulator}

The phase and amplitude imbalances of the I/Q modulator and DAC offsets can be compensated in FPGA by predistorting the driving signals before outputting them via DACs, see Fig. 8 [17].

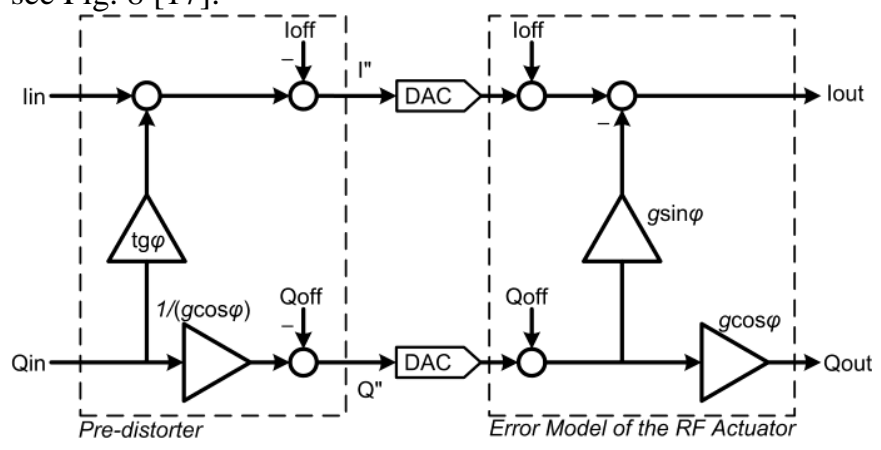

Fig. 8. Correction of the $\mathrm{I} / \mathrm{Q}$ modulator with a pre-distorter.

With the formula below, the pre-distortion matrices $A$ and $B$ are used to calculate the final DAC outputs $\left(V_{D A C}\right)$ from the desired DAC signals for an ideal RF actuator $\left(V_{i n}\right)$ to compensate the errors in the actual RF actuator:

$$
\begin{aligned}
& V_{D A C}=A V_{\text {in }}-B \\
& \text { where } \\
& V_{D A C}=\left[\begin{array}{l}
I^{\prime \prime} \\
Q^{\prime \prime}
\end{array}\right], V_{\text {in }}=\left[\begin{array}{l}
I_{\text {in }} \\
Q_{\text {in }}
\end{array}\right], \\
& A=\left[\begin{array}{cc}
1 & \operatorname{tg} \varphi \\
0 & \frac{1}{g \cos \varphi}
\end{array}\right], \quad B=\left[\begin{array}{l}
I_{\text {off }} \\
Q_{\text {off }}
\end{array}\right] .
\end{aligned}
$$

The correction can be done iteratively to converge to optimal pre-distortion matrices and adapt to the drifts of the DAC offsets and I/Q imbalances. The DAC settings can be 
scanned for a new iteration and a new set of error model parameters described in equation (4) can be calculated and then the incremental pre-distortion matrices. The new predistortion matrices of iteration $n+1$ can be calculated from the matrices of iteration $n$ and the incremental matrices:

$$
\begin{aligned}
& A_{i n c}=\left[\begin{array}{cc}
1 & \operatorname{tg} \varphi_{n} \\
0 & \frac{1}{g_{n} \cos \varphi_{n}}
\end{array}\right], \quad B_{i n c}=\left[\begin{array}{l}
I_{o f f, n} \\
Q_{o f f, n}
\end{array}\right] \\
& A_{n+1}=A_{n} A_{i n c}, \quad B_{n+1}=A_{n} B_{i n c}+B_{n} .
\end{aligned}
$$

\section{Firmware and Software for I/Q Corrections}

Six registers are defined in the FPGA firmware module for the RF actuator corrections implementing the pre-distortion functionality described in equation (5). The block diagram of the firmware module is depicted in Fig. 9. It generates a delay of one clock cycle in the Virtex-5 FPGA.

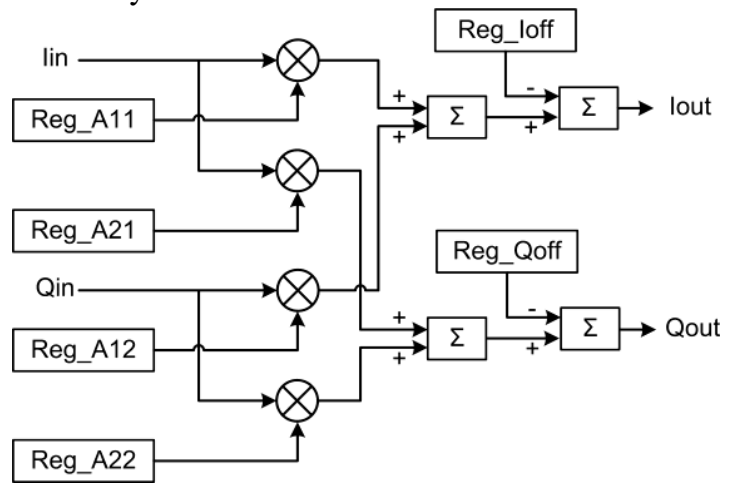

Fig. 9. Block diagram of the firmware module for RF actuator corrections.

A software module is defined in the software of the LLRF system to scan the DAC settings, read the measurement results of the output of I/Q modulator, calculate the pre-distortion matrices iteratively and set the FPGA registers.

The software should be able to calibrate the I/Q modulator without interrupting the klystron operations so that the correction can be done from time to time to adapt to the drifts of the I/Q imbalances and reference leakage. To do this, a calibration pulse is generated after the RF pulse for beam operation. The calibration pulse does not have effect on the $1 \mathrm{~kW}$ amplifier and the klystron by starting after the time window for power amplification to avoid disturbing the beam acceleration. Several phase steps are generated to rotate within the calibration pulse to perform the scanning of DAC settings for a full circle (see Fig. 10). Fig. 11 shows the waveforms after several iterations of the correction.

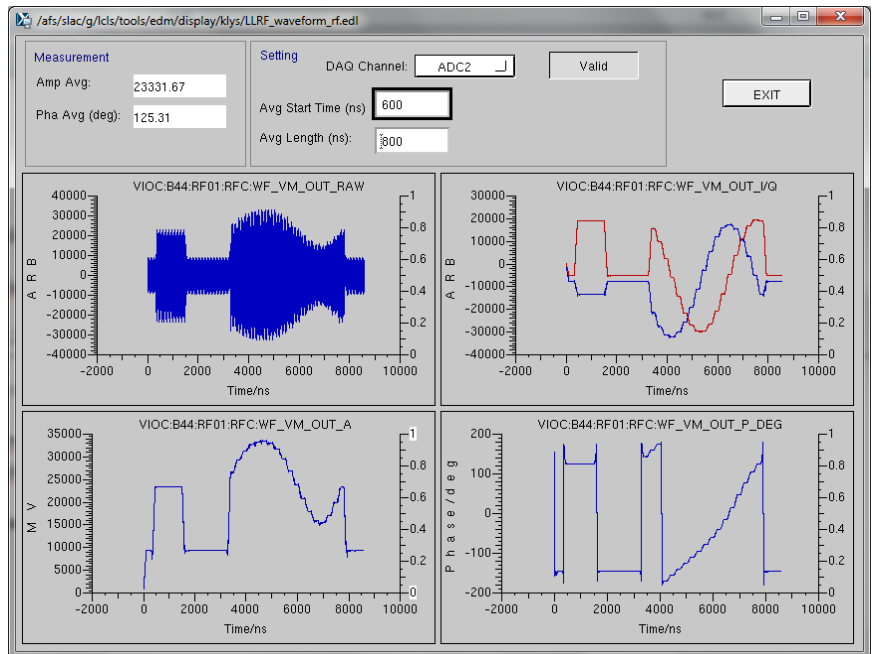

Fig. 10. Output of the $\mathrm{I} / \mathrm{Q}$ modulator showing the pulse for beam acceleration (short pulse with constant phase) and the pulse for calibration (long pulse with phase steps). The figure shows the measurement with ADC raw data (upper left), demodulated I and Q components (upper right), amplitude (bottom left) and phase (bottom right). The figure shows the waveforms before the correction and the errors on amplitude and phase are visible.

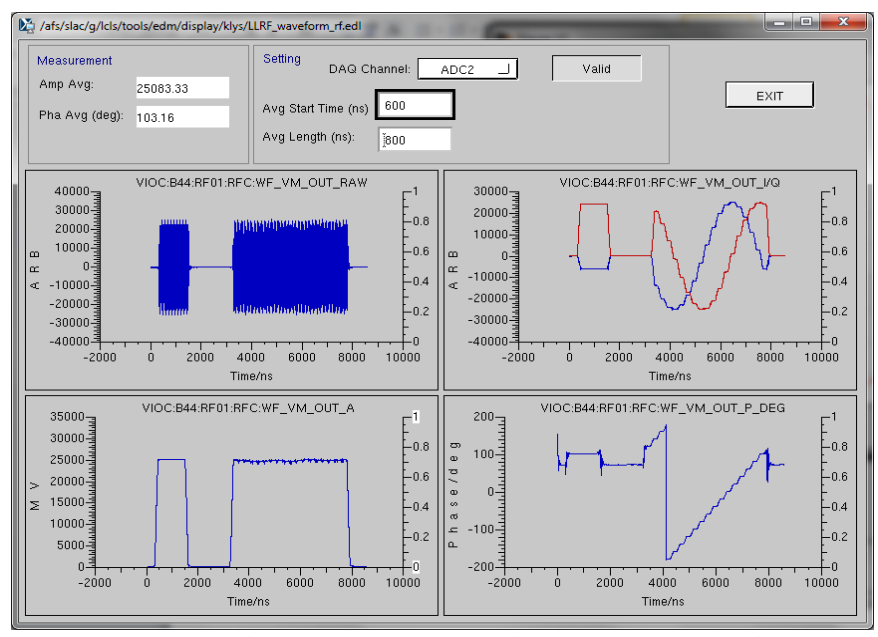

Fig. 11. Output of the I/Q modulator after correction. The flatness of amplitude and the linearity of phase are significantly improved. The residual errors are mainly caused by the imperfection of the error model.

\section{RF ACTUATOR TEST}

\section{A. Additive Noise}

The RF actuator was tested at the Linac of LCLS. The reference signal and the output of the I/Q modulator were measured by the LLRF down converters and ADCs. The RF system worked at a repetition rate of $120 \mathrm{~Hz}$ and for each RF pulse, the phase and amplitude were calculated by averaging the phase and amplitude waveforms within a time window of $0.86 \mu \mathrm{s}$ which equaled to the filling time of the accelerator structures. The measurements were limited within the bandwidth of the structures interacting with the beam by averaging in the time window. The phase and amplitude measurements for 2048 pulses are shown in Fig. 12 and the pulse-to-pulse jitters in phase and amplitude of the two signals are depicted in Table II. 

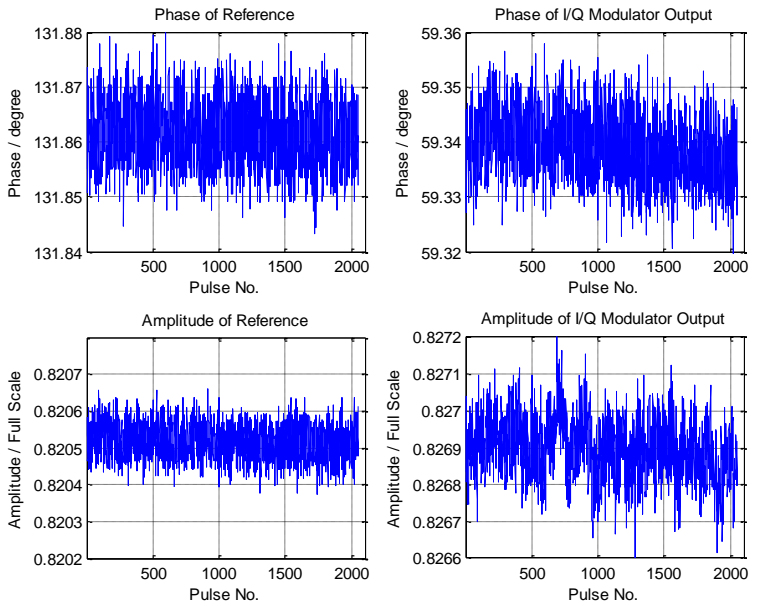

Fig. 12. Phase and amplitude measurements of the reference signal and the output of the I/Q modulator for 2048 pulses.

TABLE II

PULSE-TO-PULSE JiTTERS (RMS) MEASURED AT LCLS

\begin{tabular}{llll}
\hline \hline \multicolumn{1}{c}{ Parameter } & Reference & $\begin{array}{c}\text { Output of I/Q } \\
\text { Modulator }\end{array}$ & $\begin{array}{c}\text { Additive } \\
\text { Jitter }\end{array}$ \\
\hline Phase Jitter (degree) & 0.0057 & 0.0060 & 0.0019 \\
Amplitude Jitter (relative) & $5.77 \mathrm{e}-5$ & $1.00 \mathrm{e}-4$ & $8.17 \mathrm{e}-5$ \\
\hline \hline
\end{tabular}

The additive jitters from the RF actuator were quite below the limits, which means the hardware design of the DAC circuit and I/Q modulator and the selection of the power supply were successful.

\section{B. Carrier and Image Suppression}

If the RF actuator is used to generate a frequency offset with the SSB modulation scheme, the reference leakage will generate carrier frequency feed through and the I/Q imbalances will generate an image frequency at the output of I/Q modulator. The figure below shows that the calibration algorithm presented in this paper can suppress the feed through and image frequency signals to more than $-68 \mathrm{~dB}$ and $-52 \mathrm{~dB}$ lower than the desired signal respectively.
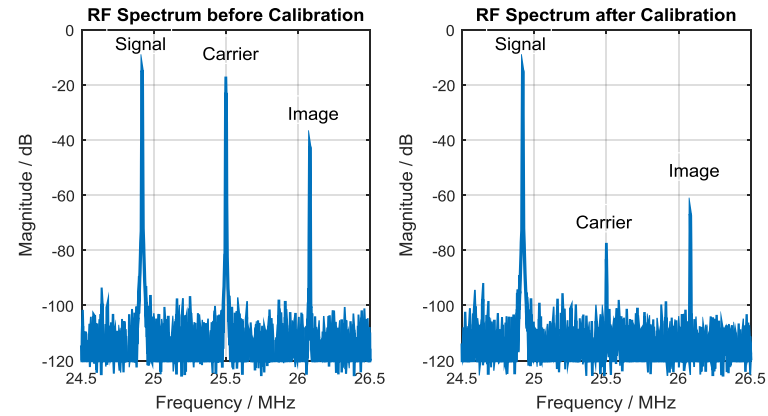

Fig. 13. Spectrum of the frequency offset signal before and after I/Q calibration. The carrier frequency is $2856 \mathrm{MHz}$ and the SSB modulation frequency is $0.58 \mathrm{MHz}$. The figure shows the spectrum of the down converted signal around the IF frequency. With calibration, the carrier feed through and image frequency signals were reduced by $-60 \mathrm{~dB}$ and $-25 \mathrm{~dB}$ respectively.

\section{RF Actuation Accuracy}

Without the calibrations of DAC offsets and I/Q imbalances, the accuracy of the phase and amplitude actuations were worse than 10 degree and $10 \%$ respectively.
The phase and amplitude actuation errors with the calibrations in Fig. 11 were evaluated in two cases. In the first case, the amplitude setting was kept constant and the phase setting was scanned for a full circle. The integrated phase actuation error was within \pm 1 degree and the amplitude actuation error was within $\pm 1 \%$ (see Fig. 14). In the second case, the phase setting was kept constant and the amplitude setting was scanned from $50 \%$ to the full scale. The phase actuation error was within \pm 1 degree and the amplitude actuation error was within $\pm 1 \%$ (see Fig. 15).
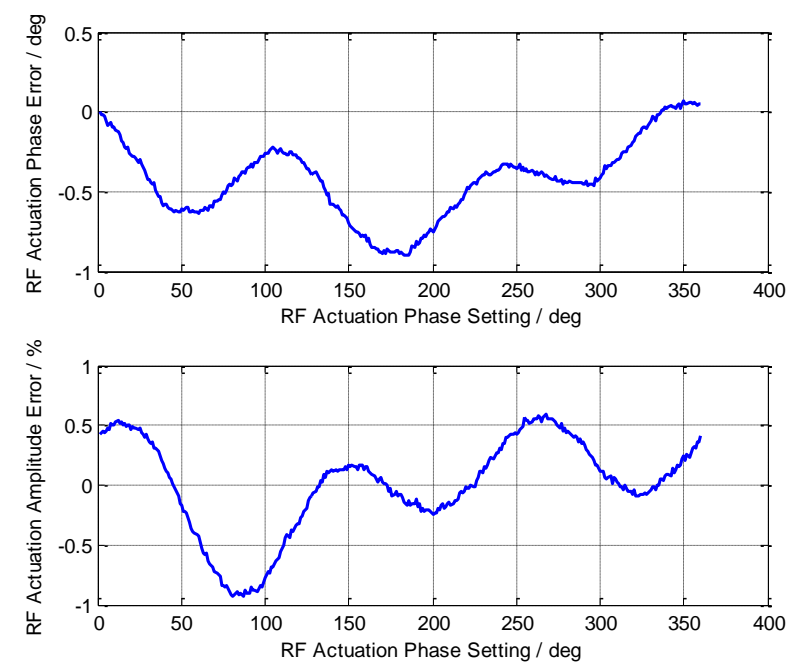

Fig. 14. Phase and amplitude actuation errors of the RF actuator after correction with amplitude setting constant and phase setting scanned for a full circle.
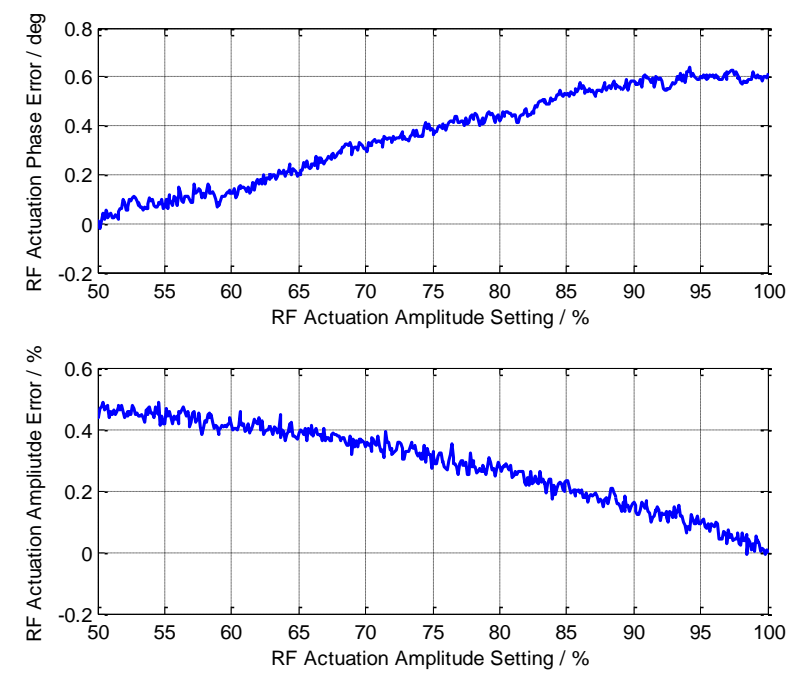

Fig. 15. Phase and amplitude actuation errors of the RF actuator after correction with phase setting constant and amplitude setting scanned from $50 \%$ to full scale. The amplitude linearity also depends on the DAC board used for providing the I/Q driving signals.

\section{CONCLUSION}

The I/Q modulator used for direct up-conversion is proved to be a robust and well working way for RF phase and amplitude actuations. The low noise hardware design minimizes the additive jitters to the RF system from the RF 
actuator. An on-line automatic calibration algorithm was developed to compensate the RF reference leakage and the I/Q imbalances without interrupting the machine operation. With the calibration, the RF actuator can provide good accuracies on phase and amplitude actuations which can well satisfy the requirements of the LCLS LLRF systems.

\section{REFERENCES}

[1] V.K. Bharadwaj, K. Bane and J. Clendenin, "Linac Design for the LCLS Project at SLAC (Published Conference Proceedings style)," in Proc. PAC97 Conf., Vancouver, B.C., Canada, 1997, pp. 903-905.

[2] C.I. Clarke, F.J. Decker and R.J. England, "FACET: SLAC's New User Facility (Published Conference Proceedings style)," in Proc. IPAC2012 Conf., New Orleans, Louisiana, USA, 2012, pp. 2741-2743.

[3] R.B. Neal, The Stanford Two-mile Accelerator. New York: W. A. Benjamin, 1968.

[4] R. Akre, "LCLS LLRF Upgrades to the SLAC Linac (Published Conference Proceedings style)," in Proc. PAC07 Conf., Albuquerque, New Mexico, USA, 2007, pp. 2421-2423.

[5] R. Boudot and E. Rubiola, "Phase Noise in RF and Microwave Amplifiers," IEEE Transactions on Ultrasonics, Ferroelectrics, and Frequency Control, vol. 59, pp. 2613-2624, Dec. 2012.

[6] P. McIntosh and R. Akre, "Overview of the RF Systems for LCLS (Published Conference Proceedings style)," in Proc. PAC05 Conf., Knoxville, Tennessee, USA, 2005, pp. 2753-2755.

[7] S.A. Tretter, Communication System Design Using DSP Algorithms. New York: Springer, 1995.

[8] V. Ayvazyan, W. Koprek and Z. Geng, "Design and Implementation of Automatic Cavity Resonance Frequency Measurement and Tuning Procedure for FLASH and European XFEL Cryogenic Modules (Published Conference Proceedings style)," in Proc. IPAC2011 Conf., San Sebastian, Spain 2011, pp. 439-441.

[9] S. Michizono, H. Katagiri and T. Matsumoto. "Performance of Digital LLRF System for STF in KEK (Published Conference Proceedings style)," in Proc. LINAC08 Conf., Victoria, BC, Canada 2008, pp. 10481050.

[10] M. Hoffmann, H. Kay and U. Mavric, "Precision LLRF Controls for the S-band Accelerator Regae (Published Conference Proceedings style)," in Proc. IPAC2013 Conf., Shanghai, China, 2013, pp. 2938-2940.

[11] P. Tosovsky and D. Valuch, "Improvement of RF Vector Modulator Performance by Feed-forward Based Calibration," Radio Engineering, vol. 19, No. 4, pp. 627-632, Dec. 2010.

[12] K.A. Santos and J.D. McVey, "Quadrature Measurement and Calibration of a Vector Modulator (Patent style)," U.S. Patent 5119399 A, June 2, 1992.

[13] H. Tseng, W. Cho and T. Chang, "Compensation of IQ Imbalance and DC Offset for OFDM Transmission over Frequency Selective Channels," IEEE International Conference on Communication, pp. 641-645, Mar. 2008.

[14] J. Tubbax and B. Come, "Compensation of IQ Imbalance and Phase Noise in OFDM Systems," IEEE Transactions on Wireless Communications, vol. 4, No. 3, pp. 872-877, May 2005.

[15] M. Hoffmann, "Development of a multichannel RF field detector for the Low-Level RF control of the Free-Electron Laser at Hamburg (Thesis or Dissertation style)," Ph.D. dissertation, Hamburg University of Technology, Hamburg, Germany, 2008.

[16] L. Doolittle. "Digital Low-Level RF Control Using Non-IQ Sampling (Published Conference Proceedings style)," in Proc. LINAC2006 Conf., Knoxville, Tennessee USA, 2006, pp. 568-570.

[17] X. Huang and M. Caron, "Gain/phase imbalance and DC offset compensation in quadrature modulators," in IEEE International Symposium on Circuits and Systems 2002, vol. 4, pp. IV-811-IV-814, 26-29 May 2002. 